\title{
A General Projection Method for the System of Relaxed Cocoercive Variational Inequalities in Hilbert Spaces
}

\author{
Changqun $\mathrm{Wu}$ \\ School of Business and Administration, Henan University, Kaifeng 475001, China \\ Meijuan Shang \\ Department of Mathematics, Shijiazhuang University, Shijiazhuang 050035, China \\ Xiaolong Qin \\ Department of Mathematics, Tianjin Polytechnic University, Tianjin 300160, China
}

The research is financed by Henan University. No. 06YBZR034.

\begin{abstract}
In this paper, we consider a new algorithm for a generalized system for relaxed cocoercive nonlinear inequalities in Hilbert spaces by the convergence of projection methods. Our results extend and improve the recent ones announced by many others.
\end{abstract}

Keywords: Relaxed cocoercive nonlinear variational inequality, Projection method, Relaxed cocoercive mapping

\section{Introduction and preliminaries}

Variational inequalities introduced by Stampacchia in the early sixties have had a great impact and influence in the development of almost all branches of pure and applied sciences and have witnessed an explosive growth in theoretical advances, algorithmic development, see [1-6] and references therein.

Let $H$ be a real Hilbert space, whose inner product and norm are denoted by $\langle\cdot, \cdot\rangle$ and $\|\bullet\|$ respectively. Let $C$ be a closed convex subset of $H$ and let $A: C \rightarrow H$ be a nonlinear mapping. Let $P_{C}$ be the projection of $H$ onto the convex subset $C$. The classical variational inequality which denoted by $\operatorname{VI}(C, A)$ is to find $u \in C$ such that

$$
\langle A u, v-u\rangle \geq 0, \forall v \in C .
$$

Recall that $A$ is said to be relaxed $(u, v)$-cocoercive if there exist two constants $u, v>0$ such that

$$
\langle A x-A y, x-y\rangle \geq(-u)\|A x-A y\|^{2}+v\|A x-A y\|^{2}, \quad \forall x, y \in C .
$$

Consider a system (SNVID) of nonlinear variational inequality problems as follows:

Find $x^{*}, y^{*}, z^{*} \in C$ such that

$$
\begin{array}{ll}
\left\langle s T_{1}\left(x^{*}, y^{*}, z^{*}\right)+x^{*}-y^{*}, x-x^{*}\right\rangle \geq 0, & \forall x \in C, s>0, \\
\left\langle t T_{2}\left(x^{*}, y^{*}, z^{*}\right)+y^{*}-z^{*}, x-y^{*}\right\rangle \geq 0, & \forall x \in C, t>0, \\
\left\langle r T_{1}\left(x^{*}, y^{*}, z^{*}\right)+z^{*}-x^{*}, x-z^{*}\right\rangle \geq 0, & \forall x \in C, r>0,
\end{array}
$$

One can easily see the SNVID problems (1.3), (1.4) and (1.5) are equivalent to the following projection formulas:

$$
\begin{array}{cc}
x^{*}=P_{C}\left[y^{*}-s T_{1}\left(y^{*}, z^{*}, x^{*}\right)\right], & s>0, \\
y^{*}=P_{C}\left[z^{*}-t T_{2}\left(z^{*}, x^{*}, y^{*}\right)\right], & t>0, \\
z^{*}=P_{C}\left[x^{*}-r T_{3}\left(x^{*}, y^{*}, z^{*}\right)\right], & r>0,
\end{array}
$$

respectively, where $P_{C}$ is the projection of $H$ onto $C$. 


\section{Algorithms}

In this section, we consider an introduction of the general three-step models for the projection methods and its special form can be applied to the convergence analysis for the projection methods in the context of the approximation solvability of the SNVID problems (1.3)-(1.5).

Algorithm 2.1. For any $x_{0}, y_{0} \in C$, , compute the sequences $\left\{x_{n}\right\},\left\{y_{n}\right\}$ and $\left\{z_{n}\right\}$ by the iterative processes:

$$
\begin{aligned}
& z_{n+1}=P_{C}\left[x_{n+1}-r T_{3}\left(x_{n+1}, y_{n+1}, z_{n}\right)\right], \\
& y_{n+1}=P_{C}\left[z_{n+1}-t T_{2}\left(z_{n+1}, x_{n+1}, y_{n}\right)\right], \\
& x_{n+1}=\left(1-\alpha_{n}\right) x_{n}+\alpha_{n} P_{C}\left[x_{n+1}-r T_{3}\left(x_{n+1}, y_{n+1}, z_{n}\right)\right],
\end{aligned}
$$

where $\left\{\alpha_{n}\right\}$ is a sequence in $[0,1]$ for all $n \geq n_{0}$.

In order to prove our main results, we need the following lemmas and definitions.

Lemma 2.1. Assume that $\left\{a_{n}\right\}$ is a sequence of nonnegative real numbers such that $a_{n+1} \leq\left(1-\lambda_{n}\right) a_{n}+b_{n}+c_{n}, \quad \forall n \geq n_{0}$,

where $n_{0}$ is some nonnegative integer, $\left\{\lambda_{n}\right\}$ is a sequence in $(0,1)$ with $\sum_{n=1}^{\infty} \lambda_{n}=\infty, b_{n}=o\left(\lambda_{n}\right)$ and $\sum_{n=0}^{\infty} c_{n}<\infty$, then $\lim _{n \rightarrow \infty} a_{n}=0$.

Definition 2.1. A mapping $T: C \times C \times C \rightarrow H$ is said to be relaxed $(u, v)$-cocoercive if there exist constants $u, v>0$ such that, for all $x, x^{\prime} \in C$,

$\left\langle T(x, y, z)-T\left(x^{\prime}, y^{\prime}, z^{\prime}\right), x-x^{\prime}\right\rangle \geq(-u)\left\|T(x, y, z)-T\left(x^{\prime}, y^{\prime}, z^{\prime}\right)\right\|^{2}+v\left\|x-x^{\prime}\right\|^{2}, \quad \forall y, y^{\prime}, z, z^{\prime} \in C$.

Definition 2.2. A mapping $T: C \times C \times C \rightarrow H$ is said to be $\mu$-Lipschitz continuous in the first variable if there exists a constant $\mu>0$ such that, for all $x, x^{\prime} \in C$,

$\left\|T(x, y, z)-T\left(x^{\prime}, y^{\prime}, z^{\prime}\right)\right\| \leq \mu\left\|x-x^{\prime}\right\|, \quad \forall y, y^{\prime}, z, z^{\prime} \in C$

\section{Main results}

Theorem3.1. Let $C$ be a closed convex subset of a real Hilbert space $H$. Let $T_{1}: C \times C \times C \rightarrow H$ be a relaxed $\left(u_{1}, v_{1}\right)$-cocoerceive and $\mu_{1}$-Lipschitz continuous mapping in the first variable, $T_{2}: C \times C \times C \rightarrow H$ be a relaxed $\left(u_{2}, v_{2}\right)$-cocoerceive and $\mu_{2}$-Lipschitz continuous mapping in the first variable, $T_{3}: C \times C \times C \rightarrow H$ be a relaxed $\left(u_{3}, v_{3}\right)$-cocoerceive and $\mu_{3}$-Lipschitz continuous mapping in the first variable. Suppose that $x^{*}, y^{*}, z^{*} \in C$ are solutions of the SNVID problems (1.3)-(1.5) and $\left\{x_{n}\right\},\left\{y_{n}\right\},\left\{z_{n}\right\}$ are the sequences generated by Algorithm 2.1. If $\left\{\alpha_{n}\right\}$ is a sequence in $[0,1]$ satisfying the following conditions:

(i) $\sum_{n=0}^{\infty} \alpha_{n}=\infty$;

(ii) $0<s, t, r<\min \left\{\frac{2\left(v_{1}-\mu_{\mu_{1}}{ }^{2}\right)}{\mu_{1}^{2}}, \frac{2\left(v_{2}-u_{2} \mu_{2}{ }^{2}\right)}{\mu_{2}{ }^{2}}, \frac{2\left(v_{3}-u_{3} \mu_{3}^{2}\right)}{\mu_{3}^{2}}\right\}$;

(iii) $\left.v_{1}-u_{1} \mu_{1}^{2}, v_{2}-u_{2} \mu_{2}^{2}, v_{3}-u_{3} \mu_{3}^{2}\right\}$;

then the sequences $\left\{x_{n}\right\},\left\{y_{n}\right\}$ and $\left\{z_{n}\right\}$ converges strongly to $x^{*}, y^{*}$ and $z^{*}$, respectively.

Proof. Since $\left(x^{*}, y^{*}, z^{*}\right)$ is the solution to the SNVID problems (1.3)-(1.5), we have

$$
\begin{array}{ll}
x^{*}=P_{C}\left[y^{*}-s T_{1}\left(y^{*}, z^{*}, x^{*}\right)\right], & s>0, \\
y^{*}=P_{C}\left[z^{*}-t T_{2}\left(z^{*}, x^{*}, y^{*}\right)\right], & t>0, \\
z^{*}=P_{C}\left[x^{*}-r T_{3}\left(x^{*}, y^{*}, z^{*}\right)\right], & r>0,
\end{array}
$$

Observing (2.1), we obtain

$\left\|x_{n+1}-x^{*}\right\|=\left\|\left(1-\alpha_{n}\right) x_{n}+\alpha_{n} P_{c}\left[y_{n}-s T_{1}\left(y_{n}, z_{n}, x_{n}\right)-x^{*}\right]\right\| \leq\left(1-\alpha_{n}\right)\left\|x_{n}-x^{*}\right\|+\alpha_{n}\left\|y_{n}-y^{*}-s\left[T_{1}\left(y_{n}, z_{n}, x_{n}\right)-T_{1}\left(y^{*}, z^{*}, x^{*}\right)\right]\right\|$.

By the assumption that $T_{1}$ is relaxed $\left(u_{1}, v_{1}\right)$-cocoercive and $\mu_{1}$-Lipschitz continuous in the first variable, we obtain $\left\|y_{n}-y^{*}-s\left[T_{1}\left(y_{n}, z_{n}, x_{n}\right)-T_{1}\left(y^{*}, z^{*}, x^{*}\right)\right]\right\|^{2}$

$=\left\|y_{n}-y^{*}\right\|-2 s\left\langle y_{n}-y^{*}, T_{1}\left(y_{n}, z_{n}, x_{n}\right)-T_{1}\left(y^{*}, z^{*}, x^{*}\right)\right\rangle+s^{2}\left\|T_{1}\left(y_{n}, z_{n}, x_{n}\right)-T_{1}\left(y^{*}, z^{*}, x^{*}\right)\right\|^{2}$

$\leq\left\|y_{n}-y^{*}\right\|+2 s u_{1} \mu_{1}^{2}\left\|y_{n}-y^{*}\right\|^{2}-2 s v_{1}\left\|y_{n}-y^{*}\right\|^{2}+s^{2} \mu_{1}^{2}\left\|y_{n}-y^{*}\right\|^{2}=\theta_{1}^{2}\left\|y_{n}-y^{*}\right\|^{2}$

Where $\theta_{1}^{2}=1+2 s u_{1} \mu_{1}^{2}-2 s v_{1}+s^{2} \mu_{1}^{2}$. From the conditions (ii) and (iii), we know $\theta_{1}<1$.

Substitute (3.2) into (3.1) yields that

$\left\|x_{n+1}-x^{*}\right\| \leq\left(1-\alpha_{n}\right)\left\|x_{n}-x^{*}\right\|+\alpha_{n} \theta_{1}\left\|y_{n}-y^{*}\right\|$

Now, we estimate

$\left\|y_{n+1}-y^{*}\right\|=\left\|P_{C}\left[z_{n+1}-t T_{2}\left(z_{n+1}, x_{n+1}, y_{n}\right)\right]-y^{*}\right\| \leq \| z_{n+1}-z^{*}-t\left[T_{2}\left(z_{n+1}, x_{n+1}, y_{n}\right)-T_{2}\left(z^{*}, x^{*}, y^{*}\right] \|\right.$ 
By the assumption that $T_{2}$ is relaxed $\left(u_{2}, v_{2}\right)$-cocoercive and $\mu_{2}$-Lipschitz continuous in the first variable, we obtain $\left\|z_{n+1}-z^{*}-t\left[T_{2}\left(z_{n+1}, x_{n+1}, y_{n}\right)-T_{2}\left(z^{*}, x^{*}, y^{*}\right)\right]\right\|^{2}$

$=\left\|z_{n+1}-z^{*}\right\|^{2}-2 t\left\langle z_{n+1}-z^{*}, T_{2}\left(z_{n+1}, x_{n+1}, y_{n}\right)-T_{2}\left(z^{*}, x^{*}, y^{*}\right)\right\rangle+t^{2}\left\|T_{2}\left(z_{n}, x_{n+1}, y_{n}\right)-T_{2}\left(z^{*}, x^{*}, y^{*}\right)\right\|^{2}$

$\leq\left\|z_{n+1}-z^{*}\right\|^{2}+2 t u_{2} \mu_{2}^{2}\left\|z_{n+1}-z^{*}\right\|^{2}-2 t v_{2}\left\|z_{n+1}-z^{*}\right\|^{2}+t^{2} \mu_{2}^{2}\left\|z_{n+1}-z^{*}\right\|^{2}=\theta_{2}^{2}\left\|z_{n+1}-z^{*}\right\|^{2}$

where $\theta_{2}^{2}=1+2 t u_{2} \mu_{2}^{2}-2 t v_{2}+t^{2} \mu_{2}^{2}$. From the conditions (ii) and (iii), we know $\theta_{2}<1$. Substituting (3.5) into (3.4) yields that $\left\|y_{n+1}-y^{*}\right\| \leq \theta_{2}\left\|z_{n+1}-z^{*}\right\|$, which implies that $\left\|y_{n}-y^{*}\right\| \leq \theta_{2}\left\|z_{n}-z^{*}\right\|$

Similarly, Substituting (3.6) into (3.3), we have

$\left\|x_{n+1}-x^{*}\right\| \leq\left(1-\alpha_{n}\right)\left\|x_{n}-x^{*}\right\|+\alpha_{n} \theta_{1} \theta_{2}\left\|z_{n}-z^{*}\right\|$

Next, we show that

$\left\|z_{n+1}-z^{*}\right\|=\left\|P_{C}\left[x_{n+1}-r T_{3}\left(x_{n+1}, y_{n+1}, z_{n}\right)\right]-z^{*}\right\| \leq \| x_{n+1}-x^{*}-r\left[T_{3}\left(x_{n+1}, y_{n+1}, z_{n}\right)-T_{3}\left(x^{*}, y^{*}, z^{*}\right] \|\right.$

By the assumption that $T_{3}$ is relaxed $\left(u_{3}, v_{3}\right)$-cocoercive and $\mu_{3}$-Lipschitz continuous in the first variable, we obtain $\left\|x_{n+1}-x^{*}-r\left[T_{3}\left(x_{n+1}, y_{n+1}, z_{n}\right)-T_{3}\left(x^{*}, y^{*}, z^{*}\right)\right]\right\|^{2}$

$=\left\|x_{n+1}-x^{*}\right\|^{2}-2 r\left\langle x_{n+1}-x^{*}, T_{3}\left(x_{n+1}, y_{n+1}, z_{n}\right)-T_{2}\left(x^{*}, y^{*}, z^{*}\right)\right\rangle+r^{2}\left\|T_{3}\left(x_{n+1}, y_{n+1}, z_{n}\right)-T_{3}\left(x^{*}, y^{*}, z^{*}\right)\right\|^{2}$

$\leq\left\|x_{n+1}-x^{*}\right\|^{2}+2 r u_{3} \mu_{3}^{2}\left\|x_{n+1}-x^{*}\right\|^{2}-2 r v_{3}\left\|x_{n+1}-x^{*}\right\|^{2}+r^{2} \mu_{3}^{2}\left\|x_{n+1}-x^{*}\right\|^{2}=\theta_{3}^{2}\left\|x_{n+1}-x^{*}\right\|^{2}$

where $\theta_{3}^{2}=1+2 r u_{3} \mu_{3}^{2}-2 r v_{3}+r^{2} \mu_{3}^{2}$.From the conditions (ii) and (iii), we know $\theta_{3}<1$. Substituting (3.9) into (3.8), we obtain $\left\|z_{n+1}-z^{*}\right\| \leq \theta_{3}\left\|x_{n+1}-x^{*}\right\|$, which implies $\left\|z_{n}-z^{*}\right\| \leq \theta_{3}\left\|x_{n}-x^{*}\right\|$

Similarly, substituting (3.10) into (3.7) yields that

$\left\|x_{n+1}-x^{*}\right\| \leq\left(1-\alpha_{n}\right)\left\|x_{n}-x^{*}\right\|+\alpha_{n} \theta_{1} \theta_{2} \theta_{3}\left\|x_{n}-x^{*}\right\| \leq\left[1-\alpha_{n}\left(1-\theta_{1} \theta_{2} \theta_{3}\right)\right]\left\|x_{n}-x^{*}\right\|$

Noticing that $\sum_{n=0}^{\infty} \alpha_{n}\left(1-\theta_{1} \theta_{2} \theta_{3}\right)=\infty$ and Applying Lemma 2.1 into (3.11), we can get

the desired conclusion easily. This completes the proof.

\section{References}

G. Stampacchia. (1964). Formes bilineaires coercivites surles ensembles convexes. Comptes rendus de I'Academie des Sciences. Paris 258. 4413-4416.

R.U. Verma. (2002). Generalized Partial Relaxed Monotonicity and Nonlinear Variational Inequalities. Int. J. Appl. Math. 9, 355-363.

R.U. Verma. (2004). Generalized System for Relaxed Cocoercive Variational Inequalities and Projection Methods. J. Optim. Theory Appl. 121, 203-210.

R.U. Verma. (2004).Generalized system for relaxed cocoercive variational inequalities and its projection methods. $J$. Optim. Theory Appl. 121, 203-210.

R.U. Verma. (2007). A -monotone nonlinear relaxed cocoercive variational inclusions. Central European J. Math. 5 (2) 386-396.

S.S. Chang, H.W. Joseph Lee \& C.K. Chan, (2007). Generalized system for relaxed cocoercive variational inequalities in Hilbert spaces. Appl. Math. Lett. 20, 329-334. 